\title{
Discussion to the paper by G. D. Hadley
}

\author{
Chairman: Dr Geoffrey Watkinson
}

Chairman. We are most grateful for this up-to-date appraisal of the methods of gastrophotography.

QUESTION. What percentage of your total series were false positives and were you ever able to pass this instrument through the pylorus? I believe the future of this instrument lies in distal viewing, not in lateral viewing, and I think the pure flexible end and biopsy are possible and $I$ think it is very probable that we shall be able to produce one in this country in the not too distant future.

HADLEY. It depends what you mean, I don't think we've falsely diagnosed any gastric ulcers. We made mistakes in the interpretation of gastric ulcers, in wrongly diagnosing a few as neoplasms. No other false positives. I don't think you can pass a fibrescope instrument through the pylorus, it is too fat. You can get the little GT5 through under X-ray screening, we've done it. But it doesn't seem possible then to insufflate the duodenal cap sufficiently to give working distance to get photographs, so we've no photographs of the duodenum. I agree that a forward-looking instrument will be an advance, and it may be possible then. I think there will still be difficulty of insufflating the duodenum sufficiently to get a viewing distance.

ChaIRman. I believe that you Dr Glanville as a radiologist have considerable experience with the gastrocamera?

Glanville. Radiologists at Chapel Allerton Hospital took up the GT5 for geriatric patients on which it is very difficult to perform barium meals. We also have the GTF. I do agree it would be much nicer if we had the GTF-A with the ambulant tip. We found that after using the GT5 for a while the GTF gave more information. We now only use the GT5 for reverse loop. I have got through a gastro-enterostomy and taken photographs of the small bowel but here you have to inflate and take the picture at the same time. Otherwise the small bowel would collapse round the lens. We are using GT5 purely in the X-ray department and are using it with image intensification and here we have the advantage that we are more sure that we are covering the whole of the stomach. It would be of advantage if we could do this every time. The exposure to radiation isn't very much with image intensification. We are now using the GTF in acute haematemesis on the ward, to try to find the source of bleeding, even before we attempt any acute barium meal studies.

MCCONNELL. I should be very interested to hear from Dr Hadley what advantage he finds, I can understand that the little blind instrument is very useful but I can't yet appreciate what advantage it is to the fibrescope to have a camera upon it. In other words, this GTF instrument: I've had it for just under a year and it's a very nice instrument. One doesn't have to focus it all the time like you do with the Hirschowitz instrument, but I personally haven't found it any great advantage to be able to take a photograph as well as looking through the instrument.

Chairman. You mean that subsequent analysis of the pictures hasn't shown you any more than you saw at the time of the endoscopy?

McConnell. The 48-hr delay while the films are developed is also very frustrating. One has almost lost interest I think, in that one has seen it with the naked eye and made one's report.

Chairman. Can you answer that Dr Hadley?

HADLEY. I can I agree there is a small technical reason why one should photograph as well and that is that the viewing angle of the fibrescope is, I think $60^{\circ}$, I can't quite remember, so you are getting a cone like $\Phi$ that. The viewing angle of the camera is $85^{\circ}$, so you are in fact photographing a lesion, an ulcer there, which we have in fact missed with a fibrescope. That's one reason. The second is that we have changed our minds occasionally, when we've all looked at the photographs, though only seldom. And the third reason of course is that it is very likely that you need something to show the surgeons who trust something they can look at very much better than a typewritten report.

QUESTION. I'd be grateful for any advice and also hear the experience that other people have had in examining patients who've had partial gastrectomy, or sub-total gastrectomy. Often I think X-ray appearances are difficult to interpret because of various procedures the surgeon may have done, particularly, if done for a growth or to decide whether or not they have a recurrence is obviously important. Now, what I find is that you've got so little stomach left that it is very difficult to inflate and hence very difficult to view, using GTF especially when you can't see the area around the oesophageal entrance anyway. Do you meet this sort of difficulty?

HADLEY. Oh yes, I think post-operative stomachs are very difficult indeed. It is very difficult to keep them insufflated, the air gets out wherever it can. Using the blind instrument it's entirely hit or miss, you are lucky sometimes. On the whole we didn't think we would be lucky at all, but we were lucky rather more times than we expected using the blind instrument. We do, I think, a little better with the GTF-A, but they are difficult. 